\title{
CRITICAL POINT BEHAVIOUR OF THE DIFFUSION LENGTH FOR RADIATIVE TRANSFER
}

\author{
I. F. GRANT ${ }^{1}$ AND B. H. J. McKELLAR ${ }^{2}$
}

(Received 24 July 1984; revised 8 December 1984)

\begin{abstract}
Critical point behaviour of the diffusion length $\gamma$ for the solutions of the radiative transfer equation deep in a homogenous medium is studied. The Legendre expansion of the medium's phase function $P(\cos \psi)$ is taken to be an infinite series and is characterized by the parameters $h_{0}, h_{1}, h_{2}, \ldots$ A characteristic equation for $\gamma$ is given in terms of an infinite continued fraction. From this equation it is shown that as any one of the $h_{n}$, say $h_{p}$, approaches zero, the others being held constant, $\gamma$ behaves as $h_{p}^{-\nu_{p}}$, where the critical exponent is found to be $\nu_{p}=\frac{1}{2}$ for all $p=0,1,2, \ldots$.
\end{abstract}

\section{Introduction}

The theory of radiative transfer finds application in astrophysics, meteorology, the physics of neutron propagation and the optics of scattering media.

This paper is concerned with the multiple scattering of monochromatic radiation by a macroscopically homogeneous medium of known scattering properties, where the radiation sources are infinitely far from the region of interest. Such a situation may be approached by taking an optically thick plane-parallel layer of the medium which is illuminated from one side only, and considering only regions far enough from the illuminated surface for the direct radiation to be dominated by the diffuse (scattered) radiation. The diffuse radiation field approaches a static relative angular distribution while decreasing in intensity exponentially with depth with a characteristic length called the "diffusion length" $\gamma$. This limiting intensity distribution can be thought of as representing the solution to the problem of the distribution of brightness over a thickly clouded sky.

\footnotetext{
${ }^{1}$ Physics (RAAF) Department, University of Melbourne, Parkville, Vic 3052, Australia

${ }^{2}$ School of Physics, University of Melbourne, Parkville, Vic 3052, Australia

(C) Copyright Australian Mathematical Society 1985, Serial-fee code 0334-2700/85
} 
The angular distribution of radiation scattered by a volume element of the medium is described by a phase function which may be expanded as a series of Legendre polynomials. The coefficients of this expansion are directly related to the parameters $h_{n}, n=0,1,2, \ldots$, defined below. As any one of the $h_{n}$, say $h_{p}$, approaches zero, the diffusion length becomes infinite. It was noted by Inonu [5] that $\gamma \rightarrow K_{0} h_{0}^{-1 / 2}$ as $h_{0} \rightarrow 0$, where $K_{0}$ is some constant. This behaviour is described in statistical mechanical terms (see e.g. Stanley [13]) by saying that $\gamma$ plays the role of a correlation length and $h_{0}$ that of an order parameter, with a critical point $h_{0}=0$ and a corresponding critical exponent of $\nu_{0}=\frac{1}{2}$ (i.e. $\gamma$ goes as $h_{0}^{-\nu_{0}}$ near $h_{0}=0$ ). The aim of this paper is to generalize this result by examining the critical point behaviour of $\gamma$ as any one $h_{p}$ vanishes, the other $h_{n}$ remaining finite.

It is found that for $\omega \leqslant 1$, where $\omega$ is the albedo for single scattering defined below, restriction of the discussion to situations where only $h_{p}$ vanishes leaves only the $h_{0} \rightarrow 0$ case, as we find that in this case $h_{p} \rightarrow 0$ implies $h_{0} \rightarrow 0$. This case is illustrated by light scattering, for which $\omega \leqslant 1$ always. For $\omega>1$ we find that $\gamma$ behaves as $h_{p}^{-v_{p}}$ as $h_{p}$ approaches the critical point $h_{p}=0$, with the critical exponent $\nu_{p}=\frac{1}{2}$, for all $p$. The scattering of neutrons is an example of this case, where $\omega$ is called the number of secondaries and may exceed unity. From the viewpoint of the theory of critical behaviour, the radiative transfer equation with $\omega>1$ offers an example of a system with an infinite number of order parameters, and may repay further investigation because of this point.

\section{The equation of radiative transfer}

To define the parameters that characterize a physical system, consider a beam of radiation incident normally on a plane layer of infinitesimal thickness $d s$. Of the energy incident on the layer, a fraction $\sigma d s$ is scattered from the beam and a fraction $\kappa d s$ experiences true absorption, i.e. is transformed into other forms of energy. The emission of secondaries in neutron scattering can be represented by a negative value of $\kappa . \sigma$ is called the scattering coefficient and $\kappa$ the coefficient of true absorption. $K=\kappa+\sigma$ is the extinction coefficient and determines the fraction of radiation removed from the direct beam. $\omega=\sigma / K$ is called the albedo for single scattering. Note that we are assuming that the whole radiation field is monochromatic, and in particular that no change in energy occurs upon scattering.

Distance into a plane layer of the medium is conveniently measured by the optical depth $\tau$, measured along a direction normal to the illuminated surface so that the unscattered intensity of a beam travelling in the positive $\tau$-direction is attenuated as $e^{-\tau}$, following Beers' Law. 
From the definition of the extinction coefficient, it is clear that the optical depth at a distance $l$ into the medium is given by

$$
\tau=\int_{0}^{l} K d s \text {. }
$$

This definition follows that of Van der Hulst [4]. Lang [7], and Paltridge and Platt [11]. However we note in passing that Chandrasekhar's [1] "normal optical thickness" for which he also uses the symbol $\tau$ is given in our notation by the complementary integral $\int_{i}^{\infty} K d s$.

Let $I(\tau, \Omega)$ be the total radiance at an optical depth $\tau$ propagating in the direction $\Omega=(\theta, \phi)$. In other words $I(\tau, \Omega) d \Omega d \sigma \cos \theta$ is the power transferred across the surface area $d \sigma$, in the solid angle $d \Omega$ from the direction $\Omega$, where $\Omega$ makes an angle $\theta$ with the surface $d \sigma$ [11]. This quantity $I$ is referred to as the specific intensity by Chandrasekhar [1].

The angular distribution of the radiation scattered by a microscopic volume is described in general by the phase function $P\left(\Omega, \Omega^{\prime}\right)$, where $\Omega=(\theta, \phi)$ and $\Omega^{\prime}=\left(\theta^{\prime}, \phi^{\prime}\right)$ are the directions of propogation of the scattered and incident radiation respectively, and the position $\tau$ direction is taken as the polar axis. The normalization is such that

$$
\int P\left(\Omega, \Omega^{\prime}\right) d \Omega^{\prime}=4 \pi,
$$

so that $P\left(\Omega, \Omega^{\prime}\right) /(4 \pi)$ is the ratio of the radiance scattered into the direction $\Omega$ to the incident radiance from the direction $\Omega^{\prime}$. For problems with unpolarized radiation, the phase function depends only on $\psi$, the angle between $\Omega$ and $\Omega^{\prime}$. Only this type of phase function will be considered in this paper and so we write it $P(\cos \psi)$.

The phase function may be expanded as a sum of Legendre polynomials of the first kind:

$$
P(\cos \psi)=\sum_{n=0}^{\infty}(2 n+1) \chi_{n} P_{n}(\cos \psi)
$$

In practical cases, e.g. Mie scattering from a sphere of radius $R$, or potential scattering of neutrons, it is in fact most convenient to define the phase function through the series (2.1), the coefficients being determined from the wave equation satisfied by the field, although it is also possible to represent the phase function as a finite sum of the square modulus of complex valued functions (scattering amplitudes) for which an integral equation exists.

In the case of scattering by a potential $V(r)$, much is known about the properties of the Legendre series (2.1). When $\int_{0}^{r} r|V(r)| d r$ exists, and the potential is bounded absolutely by $e^{\mu r}$ for large $r$, Martin [9] has shown that the infinite Legendre series for the differential cross section which is analogous to equation (2.1) converges inside an ellipse in the $z=\cos \psi$ plane with foci at $z= \pm 1$ and 
semi-major axis $1+2 \mu^{2} / k^{2}$, where $k$ is the wave number. The general convergence property of infinite Legendre series of the type (2.1) in the complex plane is that they converge uniformly and absolutely inside the ellipse

$$
\frac{x^{2}}{\left(\frac{R^{2}+1}{2 R}\right)^{2}}+\frac{y^{2}}{\left(\frac{R^{2}-1}{2 R}\right)^{2}}=1,
$$

where $z=x+i y$ and $R$ is the radius of convergence of the power series $\sum_{n=0}^{\infty}(2 n+1) \chi_{n} z^{n}[10]$. The series defines an analytic function of $z$ inside the ellipse. It follows that

$$
(2 n+1) \chi_{n} \sim R^{-n}
$$

so that, since $R>1$, in the potential scattering case $(2 n+1) \chi_{n}$ approaches zero exponentially fast as $n \rightarrow \infty$.

We are unaware of a similar detailed analysis of the Mie scattering case. However using the representation of the $a_{n}$ given by Chu and Churchill [2], one can show that, for $n+1>2 e k r$, where $r$ is the radius of the sphere and $k$ is the wave number, and $n>3$ and $e k r>1$,

$$
(2 n+1) \chi_{n} \leqslant K\left(\frac{e x}{n+1}\right)^{n-3}
$$

Here $K$ is an $n$ independent constant. This reflects the well known fact that the coefficients decrease rapidly for $n \gg 2 k r$. The parameter $R$ defining the ellipse (2.2) is given by

$$
R^{-1}=\varlimsup_{n \rightarrow \infty}(2 n+1) \chi_{n} .
$$

Thus we see that in this case the series (2.1) is absolutely and uniformly convergent in the entire $\cos \theta$ plane, and defines an analytic function of $\cos \theta$ in the whole plane.

For both potential scattering and Mie scattering the Legendre series (2.1) is absolutely and uniformly convergent on the closed real interval $(-1,1)$ in $\cos \psi$, so integration of (2.1) term by term over this interval is justified, and the coefficients $\chi_{n}$ can be expressed in terms of the phase function by

$$
\chi_{n}=\frac{1}{2} \int_{-1}^{1} P(\cos \phi) P_{n}(\cos \psi) d(\cos \psi) \text {. }
$$

One can in principle consider weaker conditions on $P(\cos \psi)$ than analyticity in an ellipse containing $(-1,1)$. For example, if for $x=\cos \psi \in(-1,1)$, the function $P(x)\left(1-x^{2}\right)^{-1 / 4}$ is summable over $(-1,1)$, the series $(2.1)$, with coefficients defined by (2.4) is convergent to $\frac{1}{2}[P(x+0)+P(x-0)]$ at every $x$ in the open set $(-1,1)$. Moreover the coefficients $\chi_{n}$ in this case are such that $(2 n+1) \chi_{n}=$ $O\left(n^{-1 / 2}\right)$ as $n \rightarrow \infty$ [3]. For our purposes it suffices to assume these weaker conditions on $P(\cos \psi)$. 
The definition of the phase function $P\left(\Omega, \Omega^{\prime}\right)$ leads immediately to the integrodifferential equation of radiative transfer $[1,6,7,11]$

$$
\mu \frac{\partial I(\tau, \Omega)}{\partial \tau}=-I(\tau, \Omega)+\frac{\omega}{4 \pi} \int P(\cos \psi) I\left(\tau, \Omega^{\prime}\right) d \Omega^{\prime},
$$

where $\mu=\cos \theta$. This is the form of the equation which does not separate the unscattered beam from the diffuse radiance. Again we note that Chandrasekhar's $\tau$ is the complement of ours, so that the right hand side of his equation of radiative transfer (his equation 63 ) is the negative of ours.

\section{The radiation field in deep layers}

The following properties of the radiation field in deep layers $(\tau \gg 1)$ come from physical considerations [12]:

(i) The role of direct (unscattered) radiation is negligible compared to that of the diffuse radiation.

(ii) The radiation intensity does not depend on azimuth.

(iii) The relative angular distribution of the intensity does not depend on optical depth.

Thus we consider only solutions of (2.2) of the form

$$
I(\tau, \mu)=f(\mu) e^{-k \tau} \text {. }
$$

Substitution of this form into (2.2) gives the integral equation

$$
(1-k \mu) f(\mu)=\frac{\omega}{4 \pi} \int P(\cos \psi) f\left(\mu^{\prime}\right) d \Omega^{\prime}
$$

which has $k$ as an eigenvalue and $f_{k}(\mu)$ as the corresponding eigenfunction. We are interested in the smallest nonnegative eigenvalue $k_{0}$ since it has been proved [4] that only the corresponding eigenfunction $f_{0}(\mu)$ (and also the eigenfunction $f_{0}(-\mu)$ corresponding to $\left.-k_{0}\right)$ is non-negative for $-1 \leqslant \mu \leqslant 1$, a necessary property of any physical solution [4]. It is $k_{0}$ which is called the inverse diffusion length. Its reciprocal is the diffusion length $\gamma$.

\section{Critical point behaviour and the diffusion length}

The eigenvalue $k_{0}$ (and hence $\gamma=k_{0}^{-1}$ ) depends only on $\omega$ and $\chi_{0}, \chi_{1}, \chi_{2}, \ldots$ Define

$$
h_{n}=(2 n+1)\left(1-\omega \chi_{n}\right) .
$$

Note that as $n \rightarrow \infty$, equation (2.4) implies that $\chi_{n} \rightarrow 0$, so that $h_{n} \rightarrow 2 n+1$. Van de Hulst [4] described how, when the Legendre series (2.1) terminates at 
$n=N$, a finite integer, the characteristic equation for $\gamma$ may be written as the continued fraction

$$
h_{0} \gamma=\frac{1}{h_{1} \gamma-\frac{4}{h_{2} \gamma-\frac{9}{\ldots \cdots \cdots}}} \frac{}{h_{N-1} \gamma-\frac{N^{2}}{h_{N} \gamma-(N+1) Q_{N+1}(\gamma) / Q_{N}(\gamma)}}
$$

where $Q_{n}(\gamma)$ is the Legendre function of the second kind of order $n$.

For a given phase function for which the Legendre series is finite with $N$ terms, we know all of the $h_{n}$, and hence we can solve the relation (4.2) for $\gamma$, choosing the largest solution if necessary. Suppose we have a situation where all of the $h_{n}$ are fixed to be non-zero. If we now let just one of them, say $h_{p}$, approach zero, then $\gamma$ will approach infinity. It was noted by Inonu [5] that $\gamma \rightarrow K_{0} h_{0}^{-1 / 2}$ as $h_{0} \rightarrow 0$ and by McKellar and Box [5] that $\gamma \rightarrow K_{1} h_{1}^{-1 / 2}$ as $h_{1} \rightarrow 0\left(K_{0}\right.$ and $K_{1}$ some constants). The behaviour of $\gamma$ as $h_{p} \rightarrow 0$ is described by the critical exponent $\nu_{p}$, where $\gamma$ goes as $h_{p}^{-\nu_{p}}$. The major result of this paper is that $\nu_{p}=\frac{1}{2}$ for all $p$.

\section{The infinite continued fraction}

We first extend (4.2) to the case of an infinite Legendre series given that none of the $h_{n}$ vanish, by replacing the right-hand side of (4.2) with an infinite continued fraction to obtain

$$
h_{0} \gamma=\frac{\frac{1}{h_{1} \gamma-4}}{h_{2} \gamma-\frac{9}{\ldots \ldots}} .
$$

It is necessary to prove that the right-hand side of (5.1) converges to $h_{0} \gamma$, given that all of the $h_{n}$ are fixed and non-zero. We do this under the restrictions that $h_{n} \sim 2 n+1$ as $n \rightarrow \infty$, which is a consequence of our assumptions about the infinite series (2.1) representing $P(\cos \psi)$ [3], and also that for a particular value of $p, h_{p}$ is small enough that the largest solution $\gamma$ of (5.1) is greater than a constant to be defined below which depends only on the fixed $h_{n}$. This is acceptable since we are only interested in the limit $\gamma \rightarrow \infty$, as $h_{p} \rightarrow 0$. 
The definition and properties of continued fractions may be found in Wall [14]. The value of a convergent infinite continued fraction is defined to be the limit of the sequence of its convergents, where a convergent is the finite continued fraction which results from truncation of the infinite fraction. Any finite continued fraction may be expressed rationally. Thus the $n$th convergent of the fraction appearing in (5.1) is

$$
\frac{\frac{1}{h_{1} \gamma-\frac{4}{\ldots \cdot \frac{n^{2}}{h_{n} \gamma}}}}{B_{n}(\gamma)},
$$

where $A_{n}(\gamma)$ and $B_{n}(\gamma)$ are polynomials in $\gamma$ given by the recurrence relations

$$
\begin{aligned}
& A_{n}=h_{n} \gamma A_{n-1}-n^{2} A_{n-2}, \\
& B_{n}=h_{n} \gamma B_{n-1}-n^{2} B_{n-2}, \quad n \geqslant 3,
\end{aligned}
$$

with the initial values

$$
\frac{A_{1}}{B_{1}}=\frac{1}{h_{1} \gamma}, \quad \frac{A_{2}}{B_{2}}=\frac{1}{h_{n} \gamma-\frac{4}{h_{2} \gamma}}=\frac{h_{2} \gamma}{h_{1} h_{2} \gamma-4} .
$$

The right-hand side of (5.1) is said to converge if the sequence $A_{n}(\gamma) / B_{n}(\gamma)$ converges, and its value is then defined to be the limit of that sequence.

Kuscer [6] defined the sequence of polynomials starting with $g_{0}(x)=1$, $g_{1}(x)=h_{0} x$ and continuing by the recurrence relation

$$
g_{n+1}(x)=\frac{1}{n+1}\left[h_{n} x g_{n}(x)-n g_{n-1}(x)\right] .
$$

Van de Hulst [2] examined the ratio

$$
r_{n}(x)=g_{n}(x) / g_{n-1}(x),
$$

defined for $n \geqslant 1$, and noted that (5.3) leads to the recurrence relation

$$
r_{n}(x)=\frac{n}{h_{n} \gamma-(n+1) r_{n+1}(x)} \text {. }
$$

It will be convenient to define the set of linear fractional transformations

$$
f_{n}(x)=\frac{n}{h_{n} \gamma-(n+1) x}, \quad n \geqslant 1 \text {. }
$$

Then (5.4a) may be written

$$
r_{n}=f_{n}\left(r_{n+1}\right)
$$

Van de Hulst took the $n \rightarrow \infty$ limit of (5.4a) to show that, under the assumption $h_{n}-2 n+1$, the limit as $n \rightarrow \infty$ of $r_{n}(\gamma)$ is $r(\gamma)$ where

$$
r(\gamma)=\gamma-\left(\gamma^{2}-1\right)^{1 / 2} \text {. }
$$


Van de Hulst wrote the characteristic equation for $\gamma$ as

$$
h_{0} \gamma=r_{1}(\gamma) \text {. }
$$

Repeated application of $(5.4 \mathrm{~b})$ to the right-hand side gives

$$
h_{0} \gamma=f_{1} f_{2} \cdots f_{n-1}\left[r_{n}(\gamma)\right], \quad n \geqslant 1 .
$$

We precede the proof of (5.1) by specifying the range of $\gamma$ for which convergence of its right-hand side will be shown.

In our consideration of (5.5), we want $f_{n}(x)$ to be continuous at $x=r_{n+1}(\gamma)$ for $n=1,2, \ldots$. If the infinite continued fraction in (5.1) is replaced by its $n$th convergent, then the $n$th degree polynomial equation

$$
h_{0} \gamma=\frac{A_{n}(\gamma)}{B_{n}(\gamma)}
$$

results, which will have at most $n$ real solutions, and hence a maximum solution $M_{n}$. The denominator of $f_{n}\left(r_{n+1}\right)$ is

$$
h_{n} \gamma-(n+1) r_{n+1}(\gamma)
$$

If (5.7) is zero then

$$
\frac{(n-2)^{2}}{h_{n-1} \gamma-\frac{n^{2}}{h_{n} \gamma-(n+1) r_{n+1}(\gamma)}}=0
$$

and (5.6) reduces to

$$
h_{0} \gamma=\frac{A_{n-2}(\gamma)}{B_{n-2}(\gamma)}
$$

Ensuring that $\gamma>M_{n-2}$ renders (5.8) false, and then (5.7) is non-zero. For $n=1$ and $n=2$, having (5.7) equal to zero would reduce (5.6) to $h_{0} \gamma=0$ and $h_{0} \gamma=\infty$ respectively, neither of which can be true since both $h_{0}$ and $\gamma$ are always finite and non-zero. Thus for any $n, \gamma>M_{n-2}$ implies that (5.7) is non-zero and hence that $f_{n}(x)$ is continuous at $x=r_{n+1}(\gamma)$.

Because of this, we restrict the discussion to the situations where $\gamma>M_{n}$ for $n=1, \ldots, p-2$, so that $f_{n}(x)$, regarded as a function of the variable $x$, is continuous at $x=r_{n+1}$ for $n=1, \ldots, p$. The discussion is also restricted so that $\gamma>3$ to ensure $0<r(\gamma)<\frac{1}{2}$ and, finally, restricted so that

$$
\gamma>\sup \left\{\frac{4 n}{\left|h_{n}\right|}\right\}, \text { for } n>p .
$$

The supremum is finite since $h_{n} \sim 2 n+1$ as $n \rightarrow \infty$. Note that these restrictions on $\gamma$ are independent of $h_{p}$, which will later tend to zero. Restriction (5.9) is necessary to establish the following result and thence the two lemmas below. 
Let $T$ be the open interval $(-1,1)$. For all $n>p$ and for all $x \in T$,

$$
\left|h_{n} \gamma-(n+1) x\right|>2 n \text {. }
$$

By the triangle inequality, the left-hand side is greater than $\left|h_{n}\right| \gamma-(n+1)|x|$ which is in turn greater than the right-hand side, since $\left|h_{n}\right| \gamma>4 n$ and $|x|<1$.

LEMMA 1. For all $n>p, f_{n}$ maps $T$ into $T$.

ProOF.

$$
\left|f_{n}(x)\right|=\frac{n}{\left|h_{n} \gamma-(n+1) x\right|}<1, \text { for } x \in T, \text { by }(5.10) .
$$

LEMMA 2. For all $n>p$, if $x$ and $y$ are two points in $T$ then $\left|f_{n}(x)-f_{n}(y)\right|<$ $\frac{1}{2}|x-y|$.

Proof. Consider any $n>p$. Inequality (5.10) implies that the denominators of both $f_{n}$ and

$$
\left|f_{n}^{\prime}(x)\right|=\frac{n(n+1)}{\left[h_{n} \gamma-(n+1) x\right]^{2}}
$$

are non-zero at all $x \in T$, and hence both $f_{n}$ and its derivative are continuous on $T$. For all $x \in T$,

$$
\begin{aligned}
\left|f_{n}^{\prime}(x)\right| & =\frac{n(n+1)}{\left[h_{n} \gamma-(n+1) x\right]^{2}} \\
& <\frac{n \cdot 2 n}{(2 n)^{2}}, \quad \text { by }(5.10) \\
& =\frac{1}{2} .
\end{aligned}
$$

Take any two points $x, y \in T$. Since $f_{n}$ is continuous and differentiable on $T$, the Mean Value Theorem may be applied to show that there exist $\xi \in(x, y)$ such that $f_{n}(x)-f_{n}(y)=f_{n}^{\prime}(\xi)(x-y)$. Since $\xi \in T$, we know that $\left|f_{n}^{\prime}(\xi)\right|<\frac{1}{2}$, and the lemma follows.

We now prove that

$$
\frac{p+1}{h_{p+1} \gamma-\frac{(p+2)^{2}}{\ldots}}
$$

converges to $r_{p+1}(\gamma)$. 
Because $r_{n} \rightarrow r$ as $n \rightarrow \infty$, we can choose $N>p$ such that $n>N$ implies $\left|r-r_{n}\right|<\frac{1}{2}$. Since $r<\frac{1}{2}$ (from the $\gamma>3$ restriction), $n>N$ implies $r_{n} T$. Also, for all $n>p$ we have $n / h_{n} \gamma \in T$, since $\gamma>4 n /\left|h_{n} \gamma\right|$ implies $\left|n / h_{n} \gamma\right|<1$.

Let $\varepsilon>0$ be given and choose $L>N$ such that $2^{-L}<\varepsilon$. We show that $n>L+p+1$ implies

$$
\left|\begin{array}{c}
\frac{n+1}{h_{p+1} \gamma-\underline{(p+2)^{2}}}-r_{p+1} \\
\cdots \cdots \\
h_{p+1} \gamma-\frac{n^{2}}{h_{n} \gamma}
\end{array}\right|<\varepsilon .
$$

Take arbitrary $n>L+p+1$. Then $n>N$ and so $r_{n+1} \in T$. Since $n / h_{n} \gamma \in T$ also, it follows that $\left|r_{n+1}-n / h_{n} \gamma\right|<2$. Since $f_{n}$ maps $T$ into $T, f_{n}\left(r_{n+1}\right) \in T$ and $f_{n}\left(n / h_{n} \gamma\right) \in T$. Hence $\left|f_{n}\left(r_{n+1}\right)-f_{n}\left(n / h_{n} \gamma\right)\right|<\frac{1}{2}\left|r_{n+1}-n / h_{n} \gamma\right|$. Repeating the argument for each of $f_{n-1}, f_{n-2}, \ldots, f_{p+1}$ gives

$$
\begin{aligned}
\left|f_{p+1} \cdots f_{n}\left(n / h_{n} \gamma\right)-f_{p+1} \cdots f_{n}\left(r_{n+1}\right)\right| & <\left(\frac{1}{2}\right)^{n-p}\left|r_{n+1}-n / h_{n} \gamma\right| \\
& <\left(\frac{1}{2}\right)^{L+1} \cdot 2<\varepsilon .
\end{aligned}
$$

But $f_{p+1} \cdots f_{n}\left(n / h_{n} \gamma\right)$ is simply the finite continued fraction in (5.12) and $f_{p+1} \cdots f_{n}\left(r_{n+1}\right)=r_{p+1}$ by repeated application of the recurrence relation (5.4b). Thus (5.12) holds and this shows the convergence of (5.11) to $r_{p+1}$ as $n \rightarrow \infty$.

Since $r_{n}=f_{n}\left(r_{n+1}\right)$, and since $f_{n}(x)$ is continuous at $r_{n+1}$ for $n=1, \ldots, p$ the composition of functions $f_{1} f_{2} \cdots f_{p}(x)$ is continuous at $r_{p+1}$ and so

$$
\lim _{n \rightarrow \infty} \frac{1}{h_{1} \gamma-\frac{4}{\ldots \cdot \frac{n^{2}}{h_{n} \gamma}}}=r_{1} .
$$

This is equivalent to (5.1), since $r_{1}=h_{0} \gamma$ and the left-hand side is by definition the value of the infinite continued fraction on the right-hand side of (5.1).

\section{The critical exponents}

Several authors have derived the critical exponent for $h_{0}$. Kuscer [6] suggested developing $\gamma^{-2}$ as a power series in $h_{0}$ by setting $g_{n}(\gamma)=0$ and taking $n$ large enough to give the desired number of terms. Thus

$$
\gamma^{-2}=h_{0} h_{1}+O\left(h_{0}^{2}\right)
$$


Inonu [5] noted that this showed $h_{0}=0$ is a critical point with exponent $\nu_{0}=1 / 2$. Similarly Sobolev [2] notes that, from the infinite continued fraction in (5.1), a power series expansion for $k_{0}=\gamma^{-1}$ can be made in terms of $h_{0}^{1 / 2}$, giving

$$
k_{0}=\left(h_{0} h_{1}\right)^{1 / 2}+O\left(h_{0}\right) \text {, }
$$

which is equivalent to (6.1). McKellar and Box [5] obtained a similar result as $h_{1} \rightarrow 0$ with $h_{0}$ fixed, showing that $h_{1}$ can also be regarded as an order parameter with a critical exponent of $\frac{1}{2}$.

The approach employed here is to use (5.1) to determine the critical exponent $\nu_{p}$, for general $p$, by examining the behaviour of

$$
h_{0} \gamma=A_{n}(\gamma) / B_{n}(\gamma) \text {, }
$$

as $\gamma \rightarrow \infty$ and $h_{p} \rightarrow 0$, and all other $h_{n}$ are held constant, as $\gamma \rightarrow \infty$ and $h_{p} \rightarrow 0$, and all other $h_{n}$ are held constant, since the right-hand side of (6.3) approaches the right-hand side of (5.1) as $n \rightarrow \infty$. Note that we must take $n \geqslant p$ before $h_{p}$ appears in equation (6.3).

Rewrite (6.3) as

$$
h_{0} \gamma B_{n}(\gamma)-A_{n}(\gamma)=0
$$

Using the recurrence relation

$$
\begin{aligned}
h_{0} \gamma B_{n}(\gamma)-A_{n}(\gamma)= & h_{n} \gamma\left[h_{0} \gamma B_{n-1}(\gamma)-A_{n-1}(\gamma)\right] \\
& -n^{2}\left[h_{0} \gamma B_{n-2}(\gamma)-A_{n-2}(\gamma)\right], \quad n \geqslant 3,
\end{aligned}
$$

which follows from the recurrence relations (5.2) for $A_{n}(\gamma)$ and $B_{n}(\gamma)$, it can be shown by induction that

$$
\begin{array}{r}
h_{0} \gamma B_{n}(\gamma)-A_{n}(\gamma)=\Pi_{n} \gamma^{n+1} \\
-\left[\frac{n^{2} \Pi_{n}}{h_{n} h_{n-1}}+(n-1)^{2} \frac{\Pi_{n}}{h_{n-1} h_{n-2}}+\cdots+\frac{\Pi_{n}}{h_{1} h_{0}}\right] \gamma^{n-1}+O\left(\gamma^{n-3}\right), \\
n \geqslant 1,
\end{array}
$$

where $\Pi_{n}=h_{0} h_{1} \cdots h_{n}$. Note that the expression $\Pi_{n} / h_{j} h_{j-1}$ does not contain a factor $h_{j}^{-1}$ or $h_{j-1}^{-1}$; it is simply a notational device for the product

$$
h_{n} h_{n-1} \cdots h_{j+1} h_{,-2} \cdots h_{1} h_{0} .
$$

Consider (6.6) only for $n>p$. Then $h_{p}$ is a factor of $\Pi_{n}$. Hence as $h_{p} \rightarrow 0$ and $\gamma \rightarrow \infty, \Pi_{n} \gamma^{n+1}$ will dominate all other terms containing at least one $h_{p}$ factor, since they will contain a lower power of $\gamma$ and no smaller power of $h_{p}$. Recall that all other $h_{j}$ are fixed, so the only other terms which need be considered are those not containing a factor $h_{p}$. Any such terms containing $\gamma^{n-1}$ will dominate any terms of lower power of $\gamma$. There are always exactly two such terms if $n>p$, namely

$$
-\left[p^{2} \frac{\Pi_{n}}{h_{p} h_{p-1}}+(p+1)^{2} \frac{\Pi_{n}}{h_{p+1} h_{p}}\right] \gamma^{n-1} .
$$


Keeping only the dominating terms of (6.4) gives, in this limit,

$$
\Pi_{n} \gamma^{n+1}-\left[p^{2} \frac{\Pi_{n}}{h_{p} h_{p-1}}+(p+1)^{2} \frac{\Pi_{n}}{h_{p+1} h_{p}}\right] \gamma^{n-1}+O\left(\gamma^{n-3}\right)=0
$$

and hence

$$
h_{p} \rightarrow\left[\frac{p^{2}}{h_{p-1}}+\frac{(p+1)^{2}}{h_{p+1}}\right] \gamma^{-2} .
$$

This result is now independent of $n$, and hence true in the $n \rightarrow \infty$ limit. So for all $p, \gamma \rightarrow K_{p} h_{p}^{-1 / 2}$ as $\gamma \rightarrow \infty$, where

$$
K_{p}=\left[\frac{p^{2}}{h_{p-1}}+\frac{(p+1)^{2}}{h_{p+1}}\right]^{1 / 2} \text {. }
$$

Thus the critical exponent is $\nu_{p}=\frac{1}{2}$, for all $p=0,1,2, \ldots$

\section{The $\omega \leqslant 1$ case}

It is shown in this section that the mathematical problem examined in this paper is restricted when applied to the class of physical problems for which $\omega \leqslant 1$. This class is important, since it includes all problems of light scattering. Scattering for which $\omega<1$ is called nonconservative, and cases for which $\omega=1$ are called conservative.

The restriction derives from the inequality [4]

$$
\left|(2 n+1) \omega \chi_{n}\right|<(2 n+1) \omega
$$

which follows from $P(\cos \psi)$ being non-negative. From this we have

$$
(2 n+1) \omega \chi_{n}<(2 n+1) \omega
$$

which is equivalent to

$$
(2 n+1)-h_{n}<(2 n+1)\left(1-h_{0}\right)
$$

using $h_{0}=1-\omega$ (implied by $\chi_{0}=1$, which follows from the equations in Section 2), and hence

$$
(2 n+1) h_{0}<h_{n} .
$$

Then for $0<\omega<1$, which implies $0<h_{0}<1$,

$$
0<h_{0}(2 n+1)<h_{n} \text {. }
$$

So for $n \geqslant 1, h_{n}$ may approach zero only if $h_{0}$ approaches zero simultaneously. If $\omega=1$ (conservative scattering), then $h_{0}=0$ and $\gamma$ is infinite. For $\omega>1$, as may occur in the neutron case, (7.1) becomes

$$
(2 n+1) h_{0}<h_{n}<2(2 n+1)-(2 n+1) h_{0} .
$$

Since $h_{0}<0$, the possibility of $h_{n}$ approaching zero independently of $h_{0}$ is admitted, thereby resulting in the behaviour of $\gamma$ described in this paper. 


\section{Conclusion}

We have shown that the critical point behaviour of the radiative transfer equation is such that it can have an infinite number of order parameters, which we have taken to be $h_{p}$. When the albedo exceeds unity it is possible for each of the order parameters to approach the critical value of zero independently, and the critical exponent for the diffusion length (which plays the role of the correlation length) is $\frac{1}{2}$ for each of these order parameters.

We have not investigated in this paper the critical behaviour as more than one of the $h_{n}$ becomes zero simultaneously. Because of the infinity of order parameters, this should be a rich field for future study.

\section{References}

[1] S. Chandrasekhar, Radiatıve transfer, (Dover Publications, New York, 1960).

[2] C-M Chu and S. W. Churchill, "Representation of the angular distribution scattered by a spherical particle", J. Opt. Soc. Amer. 45 (1955), 958-962.

[3] E. W. Hobson, The theory of spherical and ellipsoidal harmonics (Cambridge University Press 1931) Chapter VII.

[4] H. C. van de Hulst, "The spectrum of the anisotropic transfer equation", Astronom. and Astrophys. 9 (1970), 366-373.

[5] E. Inonu, "Scaling and time reversal for the linear monoenergetic Boltzmann equation", in Topics in mathematical physics (eds. H. Odabasi and O. Akyuz), (Colorado Associated University Press, 1977), 127-133.

[6] I. Kuscer, “Milne's problem for anisotropic scattering”, J. Math. Phys. 34 (1955), 256-266.

[7] K. R. Lang, Astrophysical formulae, (Springer-Verlag, Berlin 1974) Sec. 2.7.

[8] B. H. J. McKellar and M. A. Box, "The scaling group of the radiative transfer equation", $J$. Atmospheric Sci. 38 (1981), 1063-1068.

[9] A. Martin, "Analyticity in potential scattering", Progress in elementary particle and cosmic ray physics, Vol. VIII (eds. J. G. Wilson and S. A. Wonthuysen) (North-Holland Publ. Co., Amsterdam, 1965) pp. 1-66.

[10] A. Martin and F. Cheung, Analytictty properties and bounds of the scattering amplitudes (Gordon and Breach, New York, 1970). See footnote 1, p. 100.

[11] G. W. Paltridge and C. M. R. Platt, Radiative processes in meteorology and climatology (Elsevier, Amsterdam, 1976) Ch. 2 and 4.

[12] V. V. Sobolev, Radiative transfer (Van Nostrand, Princeton, N.J., 1963).

[13] H. E. Stanley, Introduction to phase transitions and critucal phenomena (Clarendon Press, Oxford, 1971).

[14] H. S. Wall, Continued fractions (Van Nostrand, New York, 1948). 